\title{
Guest editorial: Transcriptional control in myeloid cell development and related diseases
}

\author{
Tomohiko Tamura
}

Received: 23 February 2015 / Revised: 24 February 2015 / Accepted: 24 February 2015 / Published online: 10 March 2015

(C) The Japanese Society of Hematology 2015

The diverse cell types of the body exhibit distinct morphologies and functions, despite the fact that (with the exception of antigen receptor genes in lymphocytes) the genome sequence is essentially identical among all cell types in an individual. The molecular basis for this diversity is that among the approximately 22,000 genes encoded by the human genome, only a specific set is expressed in a given cell type. The differentiation of stem or progenitor cells to mature cells is a sequential process of activation, inactivation, or maintenance of the selected genes-i.e., transcriptional control-that ultimately determines cell fate. Numerous studies have shown that transcription factors and epigenetic regulators play essential roles in cell differentiation. Dysregulation of these processes can result in various diseases, including cancers, such as leukemias in the case of hematopoiesis.

Myeloid cells, e.g., granulocytes, monocytes/macrophages, dendritic cells, and mast cells, are generated from hematopoietic stem cells in the bone marrow via multipotent progenitors, common myeloid progenitors, granulocyte-monocyte progenitors, and more committed progenitors. Multiple factors have been identified as essential for the regulation of myelopoiesis. These include the transcription factors runt-related transcription factor 1 (RUNX1), PU.1, CCAAT/enhancer binding proteins $(\mathrm{C} /$ EBPs), and interferon regulatory factor-8 (IRF8), and the chromatin regulator mixed-lineage leukemia (MLL). In this issue of International Journal of Hematology, we present

T. Tamura $(\square)$

Department of Immunology, Yokohama City University Graduate School of Medicine, 3-9 Fukuura, Kanazawa-ku, Yokohama 236-0004, Japan

e-mail: tamurat@yokohama-cu.ac.jp four review articles focusing on the biology of these important transcriptional regulators.

Rapid advances in recent years in high-throughput experimental techniques, including microarrays, next-generation sequencing (such as chromatin immunoprecipitation sequencing and RNA sequencing), and high-sensitivity mass spectrometry, as well as developments in bioinformatics, have ushered in a new era in the study of transcriptional control. Comprehensive gene expression profiles and even the precise locations of transcription regulator binding and chromatin modifications throughout the genome can now be obtained. Systematic identification of the components of large protein complexes is also now achievable. Using such techniques, research into transcriptional control in myeloid cell development and related diseases has produced major advances in recent years.

In the first of the four reviews, Bonifer and colleagues provide an update on the developmental stage-specific function of RUNX1 and its important target, PU.1, during myelopoiesis from both cellular and molecular aspects. They also describe the close relationship between these two critical factors, which show reciprocal regulation of gene expression and synergistic function. Moreover, these authors summarize the known mutations and translocations involving RUNX1 or PU.1, as well as the 'addiction' to naïve RUNX1 and PU.1 in the development of acute myeloid leukemia (AML) [1].

Friedman describes the molecular basis of how $\mathrm{C} /$ $\mathrm{EBP} \alpha$ activates transcription as a homodimer and through its interactions with other bZIP transcription factors, such as AP-1, during myelopoiesis. The role of $\mathrm{C} / \mathrm{EBP} \alpha$ in the development of myeloid cells, particularly granulocytes and monocytes, and in the regulation of the cell cycle, survival, and quiescence, is highlighted. Moreover, Friedman summarizes the known mutations in $C E B P A$, the 
suppression of CEBPA expression, and the reduction of $\mathrm{C} /$ $\mathrm{EBP} \alpha$ activity in AML cases [2].

My colleagues and I review the role of IRF8 in regulating the development of myeloid cells, such as monocytes, dendritic cells, and granulocytes. We summarize its mode of action, including its cooperation with PU.1, inhibition of $\mathrm{C} / \mathrm{EBP} \alpha$ activity, and its involvement in transcription factor cascades. We further describe the antagonistic relationship between IRF8 and BCR-ABL in the pathogenesis of chronic myeloid leukemia [3].

Last, Yokoyama provides an update on the role of MLL in normal hematopoiesis and that of MLL fusion genes in AML and acute lymphoid leukemias. In particular, the molecular mechanisms of MLL fusion-dependent leukemogenesis are described in detail through categorization of MLL fusion proteins into four groups: MLL-AEP (AF4 family/ENL family/P-TEFb complex) fusion, MLL-AF10 family fusion, MLL active form mimicry, and MLL-dimerization domain fusion. The identification of various protein-protein interactions has contributed to depict distinct transcription mechanisms employed by each group [4].
These "Progress in Hematology" review articles highlight the most fundamental recent advances in our understanding of the critical transcriptional regulators, and the transcriptional networks centered on them, which underpin the regulation of myelopoiesis and the pathogenesis of leukemias.

\section{References}

1. Imperato MR, Cauchy P, Obier N, Bonifer C. The RUNX1-PU.1 axis in the control of hematopoiesis. Int J Hematol. 2015 (this issue).

2. Friedman AD. C/EBP $\alpha$ in normal and malignant myelopoiesis. Int J Hematol. 2015 (this issue).

3. Tamura T, Kurotaki D, Koizumi S. Regulation of myelopoiesis by the transcription factor IRF8. Int J Hematol. 2015 (this issue).

4. Yokoyama A. Molecular mechanisms of MLL-associated leukemia. Int J Hematol. 2015 (this issue). 